\title{
Malignant Melanoma of Anorectum: Two Case Reports
}

\author{
Binh Van Pham¹, Jae Hyun Kang ${ }^{2}$, Huynh Huu Phan ${ }^{1}$, Min Soo Cho ${ }^{2}$, Nam Kyu Kim² \\ ${ }^{1}$ Abdominal Surgery Department I, National Cancer Hospital, Hanoi, Vietnam; ${ }^{2}$ Division of Colorectal Surgery, Department of Surgery, \\ Severance Hospital, Yonsei University College of Medicine, Seoul, Korea
}

Malignant melanoma of the anorectum is a rare disorder. Patients often present with local symptoms similar to benign diseases. The prognosis is very poor, and almost all patients die because of metastases. We report 2 female patients with unremarkable histories. Both of them received previous operations before visiting our center after they were diagnosed with anorectal malignant melanoma. One case underwent abdominoperineal resection and postoperative chemotherapy. The other had been treated with ultralow anterior resection followed by immunotherapy.

Keywords: Melanoma; Anorectal; Abdominoperineal resection; Ultralow anterior resection; Wide local resection

\section{INTRODUCTION}

Melanoma is a malignant disease that develops from the pigmentcontaining cells known as melanocytes. This cancer commonly occurs in the skin, and its location at presentation differs between males and females. In women, melanoma most commonly occurs on the legs, while in men it is most common on the back [1]. Historically, melanoma of the anorectum is extremely rare, with a relative incidence of $0.5 \%$ to $4 \%$ of all malignancies in this region. The overall prognosis of anorectal melanoma is dismal, with a 10to 19-month survival after diagnosis [2]. Many retrospective studies have confirmed the poor prognosis of anorectal melanoma. In 2004, Malik et al. [3] reported a review of 19 patient records over the past 20 years who underwent operations, with a 5-year survival rate of about $21 \%$. Similar to a retrospective study by Zhang et al. [4], another study reported that the 1-, 2-, 3-, and 5-year survival rates of anorectal patients were $58 \%, 33 \%, 24 \%$, and $16 \%$, respectively, and the median survival time was 14.0 months.

Unfortunately, the management and treatment of anorectal melanoma continue to be challenging for all physicians and surgeons.

Received: Jul 19, 2019 - Revised: Nov 19, 2019 - Accepted: Jan 7, 2020 Correspondence to: Nam Kyu Kim, M.D.

Division of Colon and Rectal Surgery, Department of Surgery, Severance Hospital, Yonsei University College of Medicine, 50-1 Yonsei-ro,

Seodaemun-gu, Seoul 03722, Korea

Tel: +82-2-2228-2100, Fax: +82-2-313-8289

E-mail:namkyuk@yuhs.ac

ORCID: https://orcid.org/0000-0003-0639-5632

(C) 2021 The Korean Society of Coloproctology

This is an open-access article distributed under the terms of the Creative Commons Attribution NonCommercial License (https://creativecommons.org/licenses/by-nc/4.0) which permits unrestricted noncommercial use, distribution, and reproduction in any medium, provided the original work is properly cited.
Until now, a feasible strategy was not available, and existing strategies have not been consistent over time because of the lack of randomized clinical trials and the rarity of this condition. Operative management has been considered an effective approach although controversy still exists regarding the benefit of radiation therapy and chemotherapy. The purpose of this article is to report 2 cases of anorectal melanoma and to discuss diagnostic and surgical treatment options.

\section{CASE REPORTS}

Written informed consent was obtained for publication of this case report and accompanying images.

\section{Patient 1}

A 52-year-old female presented to a local general hospital with the chief complaint of tenesmus and continuous rectal bleeding of several months duration. She reported no history of cutaneous melanoma and denied hypertension and diabetes mellitus. A perianal visual and digital examination showed a bleeding pedunculated mass sticking out from the anus and a huge hemorrhoid at 6 oclock. A complete excision of the anal mass and hemorrhoidectomy were performed. The microscopic evaluation of the excised anal mass showed evidence of malignant melanoma with positive stains in immunohistochemistry for Human Melanoma Black 45 (HMB-45) and melan-A. The experts at the local hospital did not have much experience with anorectal melanoma, so the patient was transferred to our hospital.

Colonoscopy, computed tomography (CT) of the thorax-abdomen, and magnetic resonance imaging (MRI) of the pelvis were performed for the purpose of staging the disease. The colonos- 

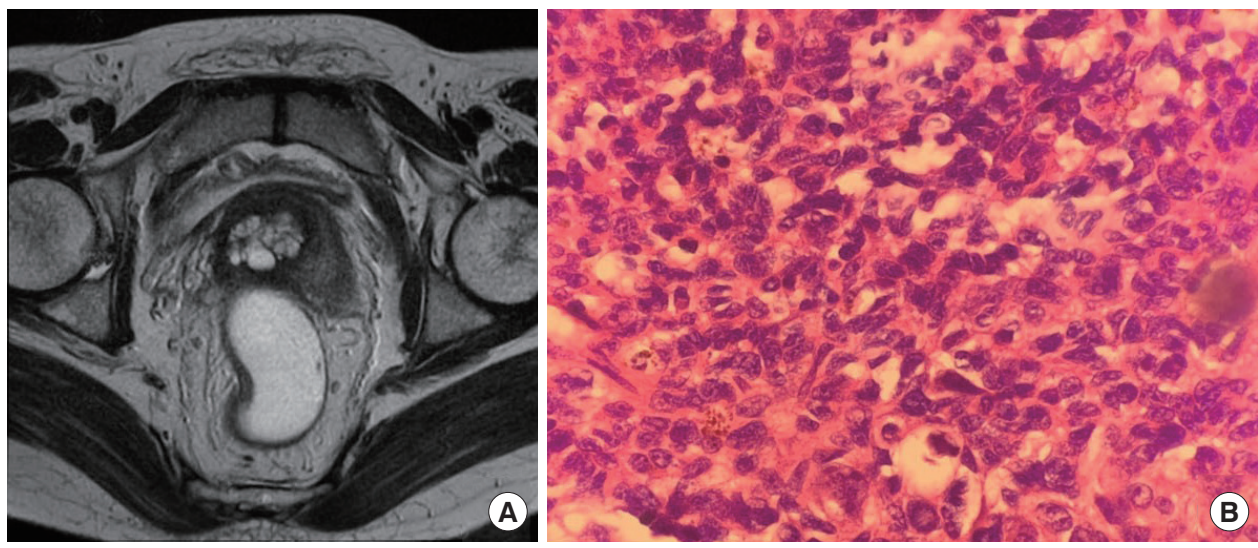

Fig. 1. Case 1. (A) Preoperative magnetic resonance imaging confirmed a well preserved anorectal fat plane and small regional lymph nodes at the perirectal space. (B) Postoperative pathological examination of a metastatic lymph node demonstrated melanin pigmentation in some cells (H\&E stain, $\times 200$ ).

copy revealed a small ulcerated area $(8 \mathrm{~mm}$ in diameter $) 1.5 \mathrm{~cm}$ from the anal verge. CT of the thorax-abdomen demonstrated no evidence of lymph nodes or distant metastases. MRI confirmed a well preserved anorectal fat plane and several small regional lymph nodes at the perirectal space (Fig. 1).

The patient was diagnosed with anal melanoma, and her case was discussed at a multidisciplinary meeting. Due to the high suspicion of regional lymph nodes metastasis and the extended length of time from the onset of initial symptoms, an abdominoperineal resection (APR) was performed. She underwent laparoscopic-assisted APR along with left-side end colostomy and tolerated the surgical procedure without any complications. Evaluation of the pathologic specimen confirmed no evidence of a malignant lesion located in the anorectal wall. One of 4 regional lymph nodes was found to be positive for metastasis. Subsequently, she received adjuvant chemotherapy with a combination of cisplatin, vinblastine, and dacarbazine. At the 6-month follow-up visit, no evidence of metastasis was observed.

\section{Patient 2}

A 39-year-old female with an unremarkable history underwent transanal excision of a polyp of the anal canal. The specimen consisted of a polypoid mass measuring $2.5 \times 2.3 \mathrm{~cm}$. The histopathological examination showed malignant melanoma and a clear resection, and the HMB-45 immunohistochemical stain result was positive. She visited our hospital for consultation about possible remnant tumor and the risk of recurrence. Therefore, we performed a series of tests to identify the stage of the disease.

Colonoscopy confirmed no evidence of residual tumor, and there was a previous operative scar at the dentate line. Five biopsies were performed, all of which were negative for anorectal carcinoma. MRI of the rectum showed 2 residual lymph node metastases in the left lateral pelvic sidewall along the internal iliac axis and 2 other lymph node metastases in the left obturator and lat- eral sacral area. No demonstrable residual primary lesions were seen in the rectum. Positron emission tomography (PET)/CT showed a focal fluorodeoxyglucose (FDG) uptake in the anal canal, which was attributed to postexcisional inflammatory change, and 2 round nodular lesions in the left perirectal area and left presacral pelvic with no discernible FDG uptake.

After consultation at a multidisciplinary team meeting, we decided that she would undergo laparoscopic ultralow anterior resection (ULAR) with coloanal anastomosis and diversion ileostomy, and she had an uncomplicated recovery. Gross examination showed a small scar located at the dentate line which corresponded to the lesion from the first transanal operation. The histopathological report showed no residual malignant melanoma and metastatic malignant melanoma in 1 out of 42 regional lymph nodes in the following location; left internal iliac (0 of 5), inferior mesenteric artery ( 0 of 4$)$, left common iliac ( 0 of 9$)$, left obturator ( 1 of 9 ) and regional ( 0 of 15). The proximal and distal margins were free from tumor. She received immunotherapy (high-dose interferon [IFN]), and 13 months after the primary operation, she underwent ileostomy repair at which time there was no evidence of recurrence.

Sixteen months after the ULAR operation, a CT of the abdomen-pelvis demonstrated metastatic lymph nodes in the aortocaval space, and radiotherapy was initiated due to the evidence of recurrence (Fig. 2).

\section{DISCUSSION}

According to the United States National Cancer Database, the skin is the most prevalent site of melanoma, although it can occur in the mouth, intestine, or eye. Mucosal melanoma is rare and accounts for approximately $1 \%$ of all melanomas. Mucosal melanomas occur mostly in the head and neck (55\%), anorectum (24\%), and vulvovaginal regions (18\%) [5]. Anorectal mucosal mela- 

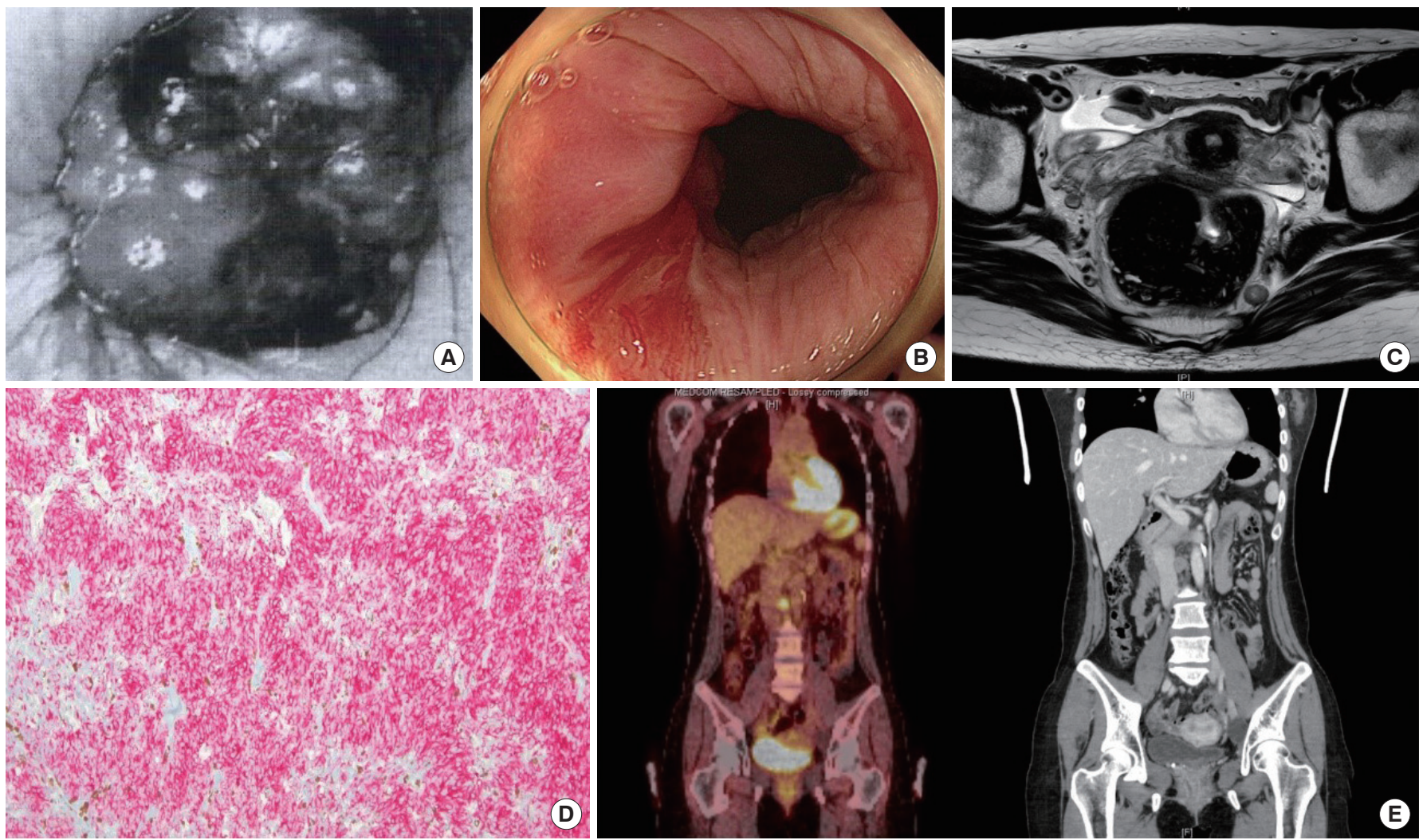

Fig. 2. Case 2. (A) Initial proctoscopy showed an anal mass. (B) Posttransanal excision colonoscopy confirmed no evidence of residual tumor. (C) Preoperative magnetic resonance imaging showed 2 residual lymph node metastases in the lateral pelvic sidewall along the internal iliac axis. (D) Postoperative pathological findings revealed that immunohistochemical staining for melanoma was positive with HMB-45 $(\times 100)$. (E) Positron emission tomography/computed tomography 16 months after ultralow anterior resection demonstrated metastatic lymph nodes in the aortocaval space.

noma accounts for $0.05 \%$ of all colorectal malignancies and $1 \%$ of all anal canal cancers [6]. The median age at diagnosis of anorectal melanoma is approximately 70 years old [7]. A study in Sweden concluded that the age-standardized incidence of anorectal melanoma was significantly higher for females (1.0 per $1,000,000$ females) than for males ( 0.7 per 1,000,000 males) throughout the 40 -year period [8]. Although several risk factors can increase the likelihood of skin melanoma, the risk factors for anorectal melanoma are not known. Epidemiologic data suggest that there is an increased risk associated with human immunodeficiency virus infection, so perhaps there is an association between anorectal melanoma and immunosuppression $[6,9]$.

The predominant local symptoms of anorectal melanoma are rectal bleeding, changed defecation pattern, and anorectal pain because $65 \%$ of anorectal melanomas are located within the anal canal or at the anal verge [6]. Unfortunately, most patients with anorectal melanoma are asymptomatic at the beginning of the disease, and if symptoms are present, they are similar to the clinical presentation of benign anorectal diseases. This similarity can cause confusion and delayed diagnosis. The first step to diagnosis is biopsy through colonoscopy or proctoscopy. However, only $34 \%$ to $41 \%$ of cases are correctly diagnosed before surgery [4, 10]. Many patients are often misdiagnosed with a benign disease, and some cases are incidental findings at pathological analysis of tissue posthemorrhoidectomy [11], adenocarcinoma, or rectal ulcer [4]. Most lesions are polypoid, with or without pigmentation, and they can be ulcerated as well [12]. One-third of anorectal melanomas are amelanotic, and given their considerable morphologic variability, misdiagnosis as lymphoma, carcinoma, or sarcoma is common [13].

Immunohistochemical staining plays an important role in the diagnosis of anorectal malignant melanoma, and S-100 proteins, melan-A, HMB-45, and tyrosinase are commonly utilized. The 2 most common melanocyte-specific stains for diagnosing malignant melanoma are antibodies to melan-A and HMB-45. These antibodies are found almost only in cells that display some form of melanocytic differentiation, because the antibodies are distinctive for premelanosome inner membrane proteins. Although these stains are very specific for melanocytic neoplasms, they have been found in other tumors with melanocytic differentia- 
tion, such as clear cell myomelanocytic tumors, lymphangioleiomyomatosis, and angiomyolipomas [12].

Imaging diagnostic studies are used for malignant diseases of the anorectum, in particular, rectal adenocarcinoma. CT scanning and ultrasonography are now important tools for staging and follow-up. In fact, endoscopy with biopsy is the best method for evaluating anorectal melanoma lesions. One study reported that CT scanning was used to diagnose primary rectal melanoma in 8 patients, and the primary rectal melanomas presented as bulky, intraluminal, fungating masses in the distal rectum focally expanding and obscuring the lumen without causing obstruction with perirectal infiltration and frequently enlarged lymph nodes [14]. Thus, CT scanning has the potential to be a useful tool in the assessment of regional diseases, especially if additional treatment methods such as chemotherapy or radiation therapy are also considered. PET can be used for staging cutaneous melanoma and may also be useful for staging anorectal melanoma. Its sensitivity was between $74 \%$ and $100 \%$ and specificity $67 \%$ in published research [15].

Neither of the patients in the present report was correctly diagnosed in the beginning. Their first diagnoses were hemorrhoid and anal mass, leading to operative procedures in the previous hospitals. After the correct diagnosis had been established, each patient underwent a radical surgery because the disease was in the advanced stage with a large lesion and evidence of lymph node metastasis on pelvic MRI. Because of the short follow-up period, only short-term results could be assessed. The patient who received the APR procedure had no complications from surgery, whereas the patient who received the ULAR experienced low anterior resection syndrome. She had severe diarrhea 10 times per day for 6 months after the diverting ileostomy repair operation. This complication decreased over time. Regrettably, 16 months after the radical operation, we discovered evidence of recurrence (periaortic lymph nodes).

Generally, surgical excision is considered as a primary treatment option for anorectal melanoma. However, standard operative procedures related to the area of resection and lymphatic dissection have not been established. There has long been a debate in the literature regarding the extent of resection necessary to optimally treat anorectal melanoma. Because the disease is so rare, randomized control trials on appropriate management are lacking, and the existing evidence is primarily a result of retrospective studies. The standard operation for anorectal melanoma is considered to be APR because it can control lymphatic spread and ensure safe resection margins for local control. Together with the advantages of APR, this technique is among the most sophisticated procedures. On the other hand, wide local excision (WLE) has clear advantages, including faster recovery, less invasive procedure, little impact on bowel function, and no need for a permanent stoma. However, it is true that the ULAR with total mesorectal clearance has been shown to be one of the most crucial approaches in treating low rectal cancer, but using ULAR for anorectal melanoma has never been reported. In theory, ULAR has the advantage of a wider excision than WLE and is equal with APR. Additionally, mesenteric lymphadenectomy could be adequately conducted by ULAR. A stellar advantage of ULAR compared to APR is preserving the gastrointestinal continuity.

An early study reported by Brady et al. [2] in 1995 suggested that aggressive treatment of the primary anorectal lesion with APR was associated with improved outcome, possibly due to regional lymphadenectomy. Among the 71 patients with resectable disease, the 5-year disease-free survival distribution of patients who underwent APR was more favorable than group who received local procedures only, although this was not statistically significant ( $27 \%$ vs. $5 \%$, respectively; $\mathrm{P}=0.11$ ). However, those who had APR were more likely to survive long term than those who did not $(\mathrm{P}<0.05)[2]$.

Yeh et al. [16] concluded in their study that there has been a gradually dramatic shift in surgical treatment toward less radical procedures. Prior to 1997, the majority of patients (15 of 21, 71\%) underwent APR. After 1997, the majority of patients (21 of 25, $84 \%)$ underwent local excision. There was no significant difference in local recurrence between patients who underwent APR vs. local excision, and the 5-year specific survival was similar [16]. A study of 251 patients with anorectal melanoma from 1960 to 1999 from the Swedish National Cancer Registry was conducted by Nilsson and Ragnarsson-Olding [17]. Sixty-six patients underwent $\mathrm{APR}$, and 86 patients underwent local excision. The median survival among the patients who were treated with either APR or local excision was 14 months, and there was no statistically significant difference in median survival rate. The overall 5-year survival rate was $11.2 \%[15,17]$.

Lymph node dissection may be indicated in clinically apparent disease or for occult disease identified with a sentinel lymph node (SLN). SLN dissection has largely been accepted as the standard of care for intermediate thickness cutaneous melanoma [18]. Experience with cutaneous melanoma has revealed a survival benefit if lymph node dissection is performed. SLN dissection has also been used with anorectal melanoma. A few case reports exist regarding the technical feasibility of sentinel node biopsy with anorectal melanoma, but the efficacy remains unknown [19]. Sentinel node biopsy may detect clinically unapparent groin adenopathy, and the subsequent inguinal dissection may provide a curative resection for a small subset of patients. It is technically feasible, but the numbers are too small to draw any meaningful conclusions at this time.

Unfortunately, there is still no guideline for systemic therapy for melanoma. Many studies consider chemotherapy and radiation therapy to be effective. Cisplatin, vinblastine, dacarbazine, IFN- $\alpha$, and interleukin- 2 are medications that may be prescribed for the treatment of this condition [20]. Another combination of chemotherapy known as the "Dartmouth regimen" uses dacarbazine, cisplatin, carmustine, and tamoxifen. A study of 240 patients showed that compared to dacarbazine alone, the Dartmouth regi- 
men increased the response rate. However, the increase was not statistically significant, and patients in both groups had a mean survival of 7 months [21]. Radiation therapy has almost no treatment effect and is mainly used in palliative care for unresectable tumors.

Recently, we have come to understand the relationship between some checkpoint proteins on $\mathrm{T}$ cells and the growth of cancer cells, for instance, PD-1/PDL-1 and CTLA-4. Malignant cells could take advantage of checkpoint proteins to develop and hide from the immune system of the body so that $\mathrm{T}$ cells cannot kill them. Humanized monoclonal antibodies, such as ipilimumab (likely blocks CTLA-4) and nivolumab and pembrolizumab (potentially block PD-1), on primed T cells have been accepted and used as standard therapies for malignant melanoma. Studies show that the response rate to inhibitor therapy is between $30 \%$ and $50 \%$ of patients, which is superior to chemotherapy [22]. However, this therapy is not applicable to all patients. Furthermore, it may lead to serious immune-related adverse events. The use of this therapy is still a challenge in some developing countries, because the cost is too high and it isn't paid for by health insurers.

In conclusion, anorectal melanoma is a rare malignant disease with a poor prognosis. Patients could be misdiagnosed as having hemorrhoids or a polyp due to the nonspecific symptoms of anal pain, rectal bleeding, or a small mass at the anorectum. Immunohistochemical staining for proteins, such as HMB-45, melan-A, and S-100, is very useful for the definite diagnosis of anorectal melanoma. Surgical resection still remains the cornerstone and most important treatment in the management of anorectal melanoma. Two surgical approaches are favored; APR and wide local resection. Until now, there have been no complete trials that demonstrated APR or WLE provide superior long-term survival.

\section{CONFLICT OF INTEREST}

No potential conflict of interest relevant to this article was reported.

\section{REFERENCES}

1. Slingluff CI Jr, Flaherty K, Rosenberg SA. Cutaneous melanoma. In: DeVita VT Jr, Lawrence TS, Rosenberg SA, editors. Cancer: principles and practice of oncology. 9th ed. Philadelphia (PA): Lippincott Williams \& Wilkins, 2011. p. 1643-91.

2. Brady MS, Kavolius JP, Quan SH. Anorectal melanoma. A 64year experience at Memorial Sloan-Kettering Cancer Center. Dis Colon Rectum 1995;38:146-51.

3. Malik A, Hull TL, Floruta C. What is the best surgical treatment for anorectal melanoma? Int J Colorectal Dis 2004;19:121-3.

4. Zhang S, Gao F, Wan D. Effect of misdiagnosis on the prognosis of anorectal malignant melanoma. J Cancer Res Clin Oncol 2010; 136:1401-5.

5. Chang AE, Karnell LH, Menck HR. The National Cancer Data
Base report on cutaneous and noncutaneous melanoma: a summary of 84,836 cases from the past decade. The American College of Surgeons Commission on Cancer and the American Cancer Society. Cancer 1998;83:1664-78.

6. Cagir B, Whiteford MH, Topham A, Rakinic J, Fry RD. Changing epidemiology of anorectal melanoma. Dis Colon Rectum 1999; 42:1203-8.

7. Iddings DM, Fleisig AJ, Chen SL, Faries MB, Morton DL. Practice patterns and outcomes for anorectal melanoma in the USA, reviewing three decades of treatment: is more extensive surgical resection beneficial in all patients? Ann Surg Oncol 2010;17:40-4.

8. Ragnarsson-Olding BK, Nilsson PJ, Olding LB, Nilsson BR. Primary ano-rectal malignant melanomas within a population-based national patient series in Sweden during 40 years. Acta Oncol 2009; 48:125-31.

9. Coté TR, Sobin LH. Primary melanomas of the esophagus and anorectum: epidemiologic comparison with melanoma of the skin. Melanoma Res 2009;19:58-60.

10. Arakawa K, Kiyomatsu T, Ishihara S, Ikemura M, Hojo D, Takiyama $\mathrm{H}$, et al. A case report of anorectal malignant melanoma with mucosal skipped lesion. Int J Surg Case Rep 2016;24:206-10.

11. van't Riet M, Giard RW, de Wilt JH, Vles W. Melanoma of the anus disguised as hemorrhoids: surgical management illustrated by a case report. Dig Dis Sci 2007;52:1745-7.

12. Chute DJ, Cousar JB, Mills SE. Anorectal malignant melanoma: morphologic and immunohistochemical features. Am J Clin Pathol 2006;126:93-100.

13. Banerjee SS, Harris M. Morphological and immunophenotypic variations in malignant melanoma. Histopathology 2000;36:387402.

14. Kim KW, Ha HK, Kim AY, Kim TK, Kim JS, Yu CS, et al. Primary malignant melanoma of the rectum: CT findings in eight patients. Radiology 2004;232:181-6.

15. Prichard RS, Hill AD, Skehan SJ, O'Higgins NJ. Positron emission tomography for staging and management of malignant melanoma. Br J Surg 2002;89:389-96.

16. Yeh JJ, Shia J, Hwu WJ, Busam KJ, Paty PB, Guillem JG, et al. The role of abdominoperineal resection as surgical therapy for anorectal melanoma. Ann Surg 2006;244:1012-7.

17. Nilsson PJ, Ragnarsson-Olding BK. Importance of clear resection margins in anorectal malignant melanoma. Br J Surg 2010;97:98103.

18. Morton DL, Thompson JF, Essner R, Elashoff R, Stern SL, Nieweg $\mathrm{OE}$, et al. Validation of the accuracy of intraoperative lymphatic mapping and sentinel lymphadenectomy for early-stage melanoma: a multicenter trial. Multicenter Selective Lymphadenectomy Trial Group. Ann Surg 1999;230:453-63.

19. Tien HY, McMasters KM, Edwards MJ, Chao C. Sentinel lymph node metastasis in anal melanoma: a case report. Int J Gastrointest Cancer 2002;32:53-6.

20. Wilson MA, Schuchter LM. Chemotherapy for melanoma. Cancer Treat Res 2016;167:209-29. 


\section{Coloproctology Binh Van Pham, et al.}

21. Chapman PB, Einhorn LH, Meyers ML, Saxman S, Destro AN, Panageas KS, et al. Phase III multicenter randomized trial of the Dartmouth regimen versus dacarbazine in patients with metastatic melanoma. J Clin Oncol 1999;17:2745-51.
22. Schachter J, Ribas A, Long GV, Arance A, Grob JJ, Mortier L, et al. Pembrolizumab versus ipilimumab for advanced melanoma: final overall survival results of a multicentre, randomised, openlabel phase 3 study (KEYNOTE-006). Lancet 2017;390:1853-62. 